\title{
Stress and disease connect at mTORC1
}

...demonstrate that the ER is wired to the mTOR pathway to control nutrient homeostasis, insulin action and survival.
Loss of the tuberous sclerosis complex (TSC) genes TSC1 and TSC2 leads to constitutive activation of the mammalian target of rapamycin complex-1 (mTORC1) and downstream signalling components, resulting in insulin resistance, the development of tumours and neurological disorders. Hotamisligil and colleagues now reveal a previously unrecognized connection between two important, highly conserved pathways - the mTORC1 and the endoplasmic reticulum (ER)-stress pathways - and demonstrate that the ER is wired to the mTOR pathway to control nutrient homeostasis, insulin action and survival.

Previous work from the Hotamisligil laboratory showed that obesity causes ER stress and promotes the development of insulin resistance, which prompted the authors to investigate whether

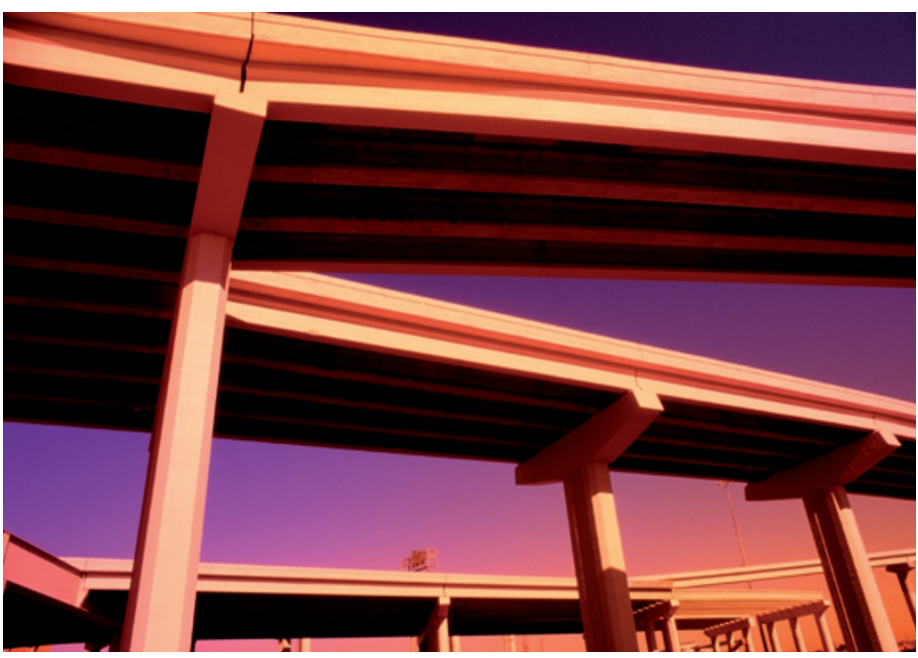

the block in insulin signalling that is associated with the loss of TSC genes might also be the result of ER stress. Indeed, both $T s c 1^{-/-}$and $T s c 2^{-/-}$ mouse embryonic fibroblasts (MEFs) showed augmentation of markers of ER stress, including increased PERK phosphorylation, increased expression of genes involved in the unfolded protein response (UPR) and activation of JNK, an important negative regulator of insulin receptor (IR) signalling. Restoration of TSC function in the TSC-knockout MEFs reversed this effect. These findings were confirmed in vivo with a TSCmutant mouse model and samples from TSC patients.

Inhibition of mTOR signalling by cycloheximide or rapamycin treatment, as well as treatment with 4-phenyl butyric acid (PBA) - a chemical chaperone that stabilizes proteins in their native conformation - suppressed the upregulation of UPR markers. This finding points to increased translation as the likely cause for UPR activation in TSC-mutant cells.

Dysregulated activation of the mTORC1 pathway triggers a negative-feedback loop that blocks IR signalling. This inhibition is most apparent in TSC-mutant cells. Treatment with PBA reversed the inhibition of IR signalling, as assessed by insulin-stimulated phospho-IRS1 (IR-substrate-1), IRS2 and phospho-AKT1 levels. Moreover, this treatment alleviated the degradation of IRS1 and IRS2 encountered in cells exposed to prolonged ER stress. These results underline the importance of ER stress as an aetiological factor in the regulation of IR inhibition.

What might be the functional significance of increased ER stress in TSC-mutant cells? Enhanced glucose deprivation induced cell death in TSC-mutant cells, which could be ameliorated by PBA treatment. Furthermore, induction of ER stress (using tunicamycin or thapsigargin treatment) led to increased apoptosis of TSC-deficient cells in a rapamycin-sensitive fashion both in vitro and in vivo. So, loss of TSC results in increased mTOR activity leading to increased ER stress, which regulates IR inhibition. In this setting, ER stress also sensitizes cells to apoptosis.

It seems that ER stress has a crucial role in the integration of metabolic and survival responses through the mTORC1 pathway. These findings have important implications for the diseases associated with mTORC1 dysregulation (obesity, diabetes and cancer), as the development of more selective and less toxic agents to trigger ER-stress pathways could offer new therapeutic opportunities, for example by selectively killing tumour cells.

Ekat Kritikou Senior Editor

Nature Reviews Molecular Cell Biology

ORIGINAL RESEARCH PAPER Ozcan, U. et al. Loss of the tuberous sclerosis complex tumor suppressors triggers the unfolded protein response to regulate insulin signaling and apoptosis. Mol. Cell 29, 541-551 (2008) FURTHER READING Reiling, J. H. \& Sabatini, D. M. Increased mTORC1 signaling UPRegulates stress. Mol. Cell 29, 533-535 (2008) 